\section{Primary hepatic vascular leiomyosarcoma of probable portal vein origin}

Primary sarcomas of the liver comprise 1-2\% of hepatic malignancies and most of these are angiosarcomas. To our knowledge only 15 cases of primary hepatic leiomyosarcoma have been reported, ${ }^{1}$ of which only two were of vascular leiomyosarcoma of the liver.

A 67 year old woman had been complaining of epigastric and right hypochondrial pain for two years. On examination she had hepatomegaly. An ultrasound scan showed a nodular vascular tumour in the left lobe of the liver and a computed tomogram showed three satellite lesions in the right lobe. All these lesions were enhanced with contrast medium in the manner of a haemangioma. At laparotomy, a large vascular tumour was found in the left lobe of the liver. Small nodules were noted in the right lobe, and a single lesion $0.8 \mathrm{~cm}$ in diameter was found in the small bowel mesentery. No lesion, however, was found in the gastrointestinal tract. A palliative left hepatectomy was performed. Her recovery was satisfactory.

The tumour measured $30 \times 15 \times 10 \mathrm{~cm}$ and weighed $1880 \mathrm{~g}$. It was well circumscribed and grey-white in appearance, with a bossellated surface. The cut surfaces had a whorled appearance and vascular spaces were evident at the periphery. The small mesenteric nodule showed similar features. Sections were stained with haemotoxylin and

\section{LETTERS TO THE EDITOR} vascular leiomyosarcomas. ${ }^{23} \mathrm{We}$ report a case eosin, elastic van Gieson, and Masson's trichrome. In addition, the unlabelled peroxidase anti-peroxidase method was used to detect desmin and vimentin (antisera from Dako Ltd), and small fragments were processed for electron microscopical examination.

The tumour and mesenteric nodule consisted of interlacing fascicles of eosinophilic spindle cells with elongated, blunt ended nuclei, and showed an intricate vascular pattern. There was pronounced nuclear pleomorphism with bizarre tumour giant cells, and numerous mitoses, many of which were abnormal. The mitotic index was $35 / 10$ high power fields. There were areas of necrosis and hyalinisation. A medium sized vein within the tumour showed pleomorphic smooth muscle cells streaming outwards from the vessel walls (figure). Bile ducts were incorporated deep within the tumour. The tumour cells stained red with Masson's trichrome and showed desmin and vimentin positivity; electron microscopical examination showed myofilaments and pinocytotic vesicles, confirming a smooth muscle origin.

The origin of parenchymal leiomyosarcomas has been discussed; most are thought to originate from connective tissue or blood vessels. In the liver the ligamentum teres has been suggested as the origin of most of the reported cases of hepatic leiomyosarcoma. ${ }^{4}$

Vascular leiomyosarcomas are rare. They arise predominantly from larger or medium sized blood vessels, the inferior vena cava accounting for over $75 \%$ of these cases. The characteristic features of these tumours include proliferating atypical smooth muscle cells, some streaming out from the media of vessels, and a striking number of neoformed blood vessels of variable size and configuration intermingled among them.

The two cases described in the liver were both of hepatic vein origin, and both presented with the Budd-Chiari syndrome. ${ }^{23}$ Our case had no evidence of the Budd-Chiari syndrome. In addition, bile ducts were present, a feature not previously described within hepatic leiomyosarcomas. A medium sized vein showed pleomorphic tumour cells streaming out from the media. The combination of these three features all suggest origin from a portal vein.

In a detailed description of vascular leiomyosarcomas ${ }^{5}$ it was concluded that the mitotic index was the most important pathological feature on which a prognostic evaluation for a vascular leiomyosarcoma could be based. Three of the patients with a mitotic count of greater than $35 / 10$ high power fields had a poor prognosis. Our case had a mitotic index of $35 / 10$ high power fields, an indication of poor prognosis, and metastases were present.

$$
\begin{array}{r}
\text { M SUNDARESAN, } \\
\text { SB KELLY, } \\
\text { IS BENJAMIN, } \\
\text { AB AKOSA } \\
\text { Departments of Histopathology and } \\
\text { Hepatobiliary Surgery, } \\
\text { Royal Postgraduate Medical School, } \\
\text { Hammersmith Hospital, } \\
\text { Du Cane Road, } \\
\text { London W12 } 0 H S
\end{array}
$$

1 Chen KTK. Hepatic leiomyosarcomas. J Surg Oncol 1983;24:325-8.

2 Koberle F, Pfleger R. Lebervenengeschwulst mit dem Symptomenbild einer Endophlebitis Obliterans Hepatica. Wein Arch Inn Med 1940;34:73-85.

3 MacMahon HE, Ball HG. Leiomyosarcomas of the hepatic vein and the Budd-Chiari synthe hepatic vein and the Budd-Chiari syn-
drome. Gastroenterology 1971;61:239-43.

4 MacSween RNM, Anthony PP, Sheuer PJ. Tumours and tumour like conditions of the
Tumen liver and biliary tract. Pathology of the liver. London: Churchill Livingstone, 1987 574-645.

5 Varela-Duran J, Oliva H, Rosai J. Vascular leiomyosarcoma. Cancer 1979;44:1684-91.

Medium sized vein in the tumour with pleomorphic tumour cells streaming out of the media (arrows).

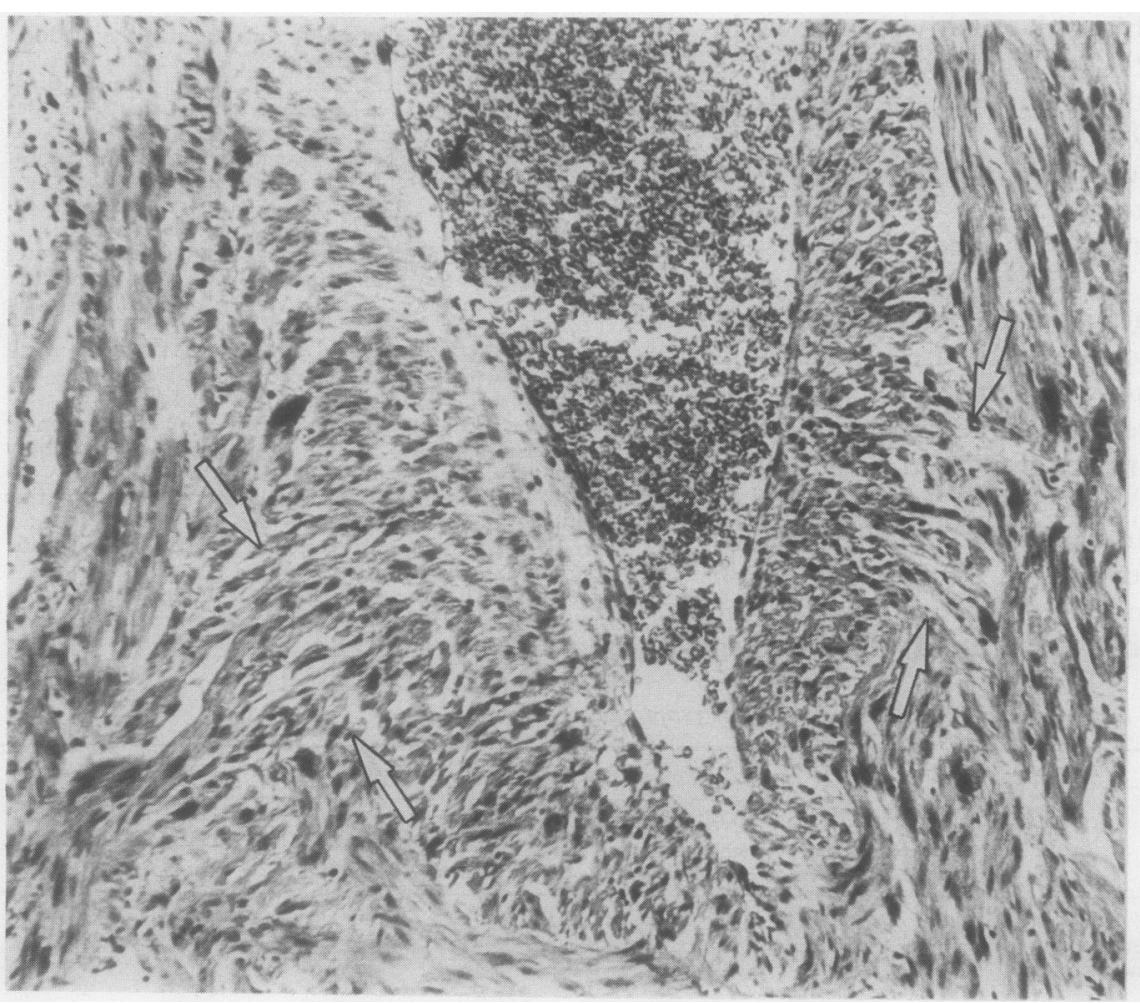

\section{Peliosis hepatis after liver transplantation}

Peliosis hepatis is characterised by bloodfilled spaces in the liver. Causes and associations identified to date include steroid hormone treatment, wasting diseases, cancer, human immunodeficiency virus disease and renal transplantation..$^{1-3}$ We report peliosis in a liver transplant recipient; as far as we are aware, this has not been previously recorded.

\section{Case report}

A 25 year old female factory employee developed acute hepatic failure following an overdose of paracetamol. Emergency orthotopic liver transplantation was carried out four days after the overdose. There was mismatching of blood groups (donor blood group A, recipient group O). A protocol baseline liver biopsy specimen taken at the time of revascularisation of the graft showed substantially normal liver. Four days later a second biopsy was performed because of rising serum aspartate transaminase activities. This showed no evidence of cellular rejection. Scattered acidophil bodies and increased numbers of liver cell mitoses were noted. A further biopsy specimen on day 7 showed, in addition to the above changes, portal infiltration, endotheliitis affecting portal venules and minor bile duct changes. The picture was interpreted as cellular rejection. 